\title{
Genome-wide copy number profiling using a 100K SNP array reveals novel disease-related genes BORIS and TSHZ1 in juvenile angiofibroma
}

\author{
BERNHARD SCHICK $^{1}$, SILKE WEMMERT ${ }^{1}$, VIVIENNE WILLNECKER ${ }^{1}$, JULIA DLUGAICZYK ${ }^{1}$, \\ PIERO NICOLAI $^{2}$, HENRYK SIWIEC $^{3}$, CHRISTIAN T. THIEL $^{4}$, ANITA RAUCH $^{4,5}$ and OLAF WENDLER ${ }^{6}$ \\ ${ }^{1}$ Department of Otorhinolaryngology, Saarland University Medical Center, Homburg/Saar, Germany; ${ }^{2}$ Department \\ of Otorhinolaryngology, University of Brescia, Italy; ${ }^{3}$ Department of Otolaryngology, Head and Neck Surgery, \\ University of Lublin, Poland; ${ }^{4}$ Institute of Human Genetics, University Erlangen-Nuremberg, Erlangen, \\ Germany; ${ }^{5}$ Institute of Medical Genetics, University of Zurich, Schwerzenbach-Zurich, Switzerland; ${ }^{6}$ Department \\ of Otorhinolaryngology, Head and Neck Surgery, Erlangen University Hospital, Erlangen, Germany
}

Received May 9, 2011; Accepted June 20, 2011

DOI: $10.3892 /$ ijo.2011.1166

\begin{abstract}
Juvenile angiofibroma (JA) is a unique fibrovascular tumor, which is almost exclusively found in the posterior nasal cavity of adolescent males. Although histologically classified as benign, the tumor often shows an aggressive growth pattern and has been associated with chromosomal imbalances, amplification of oncogenes and epigenetic dysregulation. We present the first genome-wide profiling of JAs $(n=14)$ with a 100K single nucleotide polymorphism (SNP) microarray. Among the 30 novel JA-specific amplifications detected on autosomal chromosomes with this technique, the genes encoding the cancer-testis antigen BORIS (brother of the regulator of imprinted sites) and the developmental regulator protein TSHZ1 (teashirt zinc finger homeobox 1) were selected for further analysis. Gains for both BORIS (20q13.3) and TSHZ1 (18q22.3) were confirmed by quantitative genomic PCR. Furthermore, quantitative RT-PCR revealed a significant up-regulation of BORIS $(\mathrm{p}<0.001)$ and TSHZ1 transcripts $(\mathrm{p}<0.05)$ for JAs compared to nasal mucosa. Following detection of BORIS and TSHZ1 proteins in Western blots of JAs, subcellular localization was determined for both proteins in immunostaining of JA cryosections. In conclusion, genomic copy number profiling using an SNP microarray has been proven to be a suitable and reliable tool for identifying novel disease-related genes in JAs and newly implicates BORIS and TSHZ1 overexpression in the pathogenesis of JAs. Detection
\end{abstract}

Correspondence to: Dr Bernhard Schick, Department of Otorhinolaryngology, Saarland University Medical Center, D-66421 Homburg, Germany

E-mail: bernhard.schick@uks.eu

Key words: juvenile angiofibroma, SNP array, brother of the regulator of imprinted sites, teashirt zinc finger homeobox 1 of BORIS in JAs is described with special regard to tumor proliferation and epigenetic dysregulation, and the finding of TSHZ1 amplifications is discussed with special respect to the hypothesis of JAs as malformations of the first branchial arch artery.

\section{Introduction}

Juvenile angiofibroma (JA) is an uncommon fibrovascular tumor arising almost exclusively in adolescent males close to the sphenopalatine foramen in the posterior nasal cavity. Blood supply of this hypervascular tumor is usually derived from the maxillary/sphenopalatine artery, however, the internal carotid artery must also be considered as a potential tumor feeder (1). Tumor architecture is characterized by numerous irregularly configured vessels set in a fibrous stroma. Even though this tumor is regarded to be histologically benign, an aggressive growth pattern is frequently observed (2).

Localization and blood supply have led to the hypothesis of JAs as vascular malformations (VM) arising from remnants of the first branchial arch artery $(3,4)$. Recently, this hypothesis has been supported on the molecular level by detection of laminin $\alpha 2$, which is a marker of early angiogenesis, in the perivascular extracellular matrix of JAs (5). However, there have been no detailed investigations into the expression of regulatory factors associated with the development of derivates from the first branchial arch in juvenile angiofibromas. In this context, TSHZ1 (teashirt zinc finger homeobox 1), whose ortholog has been detected in neural crest-derived mesenchymal cells of the first and second branchial arches in mice $(6,7)$, would be an attractive candidate to shed light on the tumor origin of JAs.

The increased incidence of JAs in patients with familial adenomatous polyposis coli (APC) prompted the first genetic studies on JAs (8) and suggested an involvement of the Wnt pathway. APC is part of the $\beta$-catenin destruction complex in the Wnt signaling cascade. Activation of the 
Wnt pathway inhibits the APC protein, thus increasing the cytoplasmatic pool of $\beta$-catenin, which is then translocated to the nucleus, where it acts as a transcriptional co-activator (9). Although alterations of the $A P C$ gene were not found in JAs (8), activating $\beta$-catenin gene $(C T N N B 1)$ mutations were frequently detected (10). Translocation of $\beta$-catenin into the cell nucleus together with androgen receptors (AR) and subsequent activation of AR dependent genes has been suggested to contribute to the preponderance of the tumor in male adolescents (11).

Following these initial mutation studies, comparative genomic hybridization (CGH) and fluorescence in situ hybridization (FISH) have been established as valuable genetic tools in JA research (12). In our previous study, numerous chromosomal imbalances were detected in JAs by CGH and FISH $(11,13,14)$. In this context, the observed amplification of AURKA (aurora kinase A) and MDM2 (Mdm2 p53 binding protein homolog) genes is of special interest, as both are discussed to play a role in chromosomal instability in cancers (15). Additionally, FISH revealed gains of the MYC oncogene in advanced tumor stages (16). Besides these genetic alterations associated with cell proliferation, loss of imprinting (LOI) has also been observed in JAs (17) indicating a role of epigenetic dysregulation in this rare fibrovascular tumor.

Epigenetic dysregulation is a common feature in human cancers. In recent years, the transcription factor CTCF (CCCTC-binding factor) and its paralog BORIS (brother of the regulator of imprinted sites) have been discussed as key players in this context (18). CTCF is a ubiquitously expressed, highly conserved multivalent transcription factor. Apart from controling cell proliferation-associated genes, this methylationsensitive DNA binding-protein is also involved in epigenetic regulation (19). Normally, the expression of CTCF and BORIS is mutually exclusive with BORIS almost completely restricted to spermatocytes in the male testis where it controls imprinting in the male germ line development (20). However, an aberrant expression of BORIS has been detected in various cancer cell lines and native tumors, where it is supposed to interfere with the normal regulatory functions of CTCF (18). Therefore, BORIS is regarded as a cancer-testis antigen (CTA) (21).

To the best of our knowledge, genetic alterations of either BORIS or TSHZ1 have not been analyzed in tumors using single nucleotide polymorphism (SNP) oligonucleotide microarrays to date. Due to the increased resolution of SNPs compared to $\mathrm{CGH}$, this technique of molecular karyotyping has gained growing importance in the genome-wide analysis of genetic alterations in recent years including screening for tumor-susceptibility genes (22).

We report the first application of array-CGH in JAs revealing gains in TSHZ1 and BORIS genes as a common finding. These observations were confirmed by increased levels of both TSHZ1 and BORIS mRNA in JA tissue compared to nasal mucosa and detection of TSHZ1 and BORIS proteins in JAs. These novel results are discussed in the context of JA tumor origin, tumor proliferation and epigenetic dysregulation.

\section{Materials and methods}

Tissue and blood samples. Two different sets of tissue and blood samples were used in this study. Set I comprised 14 JA tissue specimens, peripheral blood from 2 of the 14 JA patients and from 6 healthy controls. These samples were objected to molecular karyotyping using a 100K SNP array and genomic PCR analysis. Due to limited amounts of tumor tissue from set I, a different set of samples (set II) including 15 JAs and 11 nasal mucosa (NM) specimens was used for comparative BORIS and TSHZ1 mRNA/protein expression studies.

All JAs analyzed in this study were-snap frozen immediately after tumor removal. Tumor stages ranged from stage II to stage IIIb according to Andrews (23). Beside the characteristic clinical findings, diagnosis was confirmed by detailed histological and immunohistological studies, as previously reported (16). Nasal mucosa used as control tissue was derived from inferior turbinate surgery for treatment of nasal obstruction in otherwise healthy individuals. For transcript analysis, the superficial epithelial layer of the NM was removed in order to obtain a homogeneous stromal tissue sample comparable to the texture of JAs. All patients were informed and gave their written consent.

$100 K$ SNP array. DNA (500 ng) from each of the 14 JA tumor specimens and from two blood samples of affected patients (set I) was processed and analyzed with the Affymetrix GeneChip ${ }^{\circledR}$ Human Mapping 100K array (Affymetrix, Santa Clara, CA, USA), as previously described (24). Hybridization and data acquisition were performed by the Gene Mapping Center, Max-Delbrück-Center for Molecular Medicine in Berlin-Buch (Germany). Call rates exceeded $98 \%$ in all analyzed samples. Raw data were processed with the Affymetrix copy number analysis tool (CNAT) using $0.5 \mathrm{Mb}$ sliding windows for the genomic smoothening algorithm (GSA). The CNAT copy number output was exported to a table, which was then filtered for clusters of SNPs fulfilling certain criteria using the Copy Number Variation Finder (CNVF) (24). Using this software tool, copy number data were filtered for blocks fulfilling the following conditions: GSA p-values for duplications $>+2.5$ (at least 3 SNPs with a value $>+5$ ) and for deletions $<-2.5$ (at least 3 SNPs with a value <-5). The Y-chromosome was not covered by the used SNP array.

\section{Quantitative PCR}

a) Genomic PCR. Sufficient DNA for quantitative (q) genomic PCR was available from 12 JAs and 6 blood samples from healthy controls (set I). Before performing TaqMan ${ }^{\circledR}$ gene expression assays (Applied Biosystems, Darmstadt, Germany) and data analysis as previously described (25), optimal concentrations of primers, probes and templates were determined on separate multiplexed optimizing pre-runs. Primer Express 2.0 software was used to design the TaqMan primers and probes from GenBank sequences no. NM_005786 (TSHZ1) and no. NM_080618 (BORIS). Primer sequences were as follows: TSHZ1 forward, GCAAGAGACCGCGGAACAT; TSHZ1 reverse, GCTCAGGCCAGGCCTAGTG; TSHZ1 probe, CACTAAACGTCGTCGAGC; BORIS forward, ACGAGTTCTCCACTGGTGACAA, BORIS reverse, GGAACCAGGCCCTACAAGTGT and BORIS probe, TGCCATGTTGCAGTCG). For determination of gene copy numbers, the $\Delta \Delta \mathrm{Ct}$ method based on threshold cycle numbers (Ct) was employed. Our previous study showed that a $\Delta \Delta \mathrm{Ct}$ ratio $>1.3$ identified $100 \%$ of gene amplifications in blood 
samples from patients with a PMP22 duplication (26), whereas $\Delta \Delta \mathrm{Ct}$ values from 0.8 to 1.2 confirmed a diploid (normal) genotype. Due to the contamination of JAs with normal DNA from non-tumor cells, the exact $\Delta \Delta \mathrm{Ct}$ value for gains is uncertain. Considering the heterogeneous cellular composition of JAs with at least $30 \%$ inflammatory cells (27), a modified $\Delta \Delta \mathrm{Ct}$ ratio $>1.2$ for a sample was judged as an indicator for gene duplications in this study.

b) Reverse transcriptase (RT) PCR. Total RNA was isolated from the $15 \mathrm{JAs}$ and 11 nasal mucosa samples from set II using illustra ${ }^{\mathrm{TM}}$ RNAspin (GE-Healthcare, Buckinghamshire, UK) according to the manufacturer's instructions, including genomic DNA digestion. Total RNA $(1 \mu \mathrm{g})$, determined by photometrical analysis, was used for reverse transcription (QuantiTect RT-Kit, Qiagen, Hilden, Germany), obtaining $20 \mu \mathrm{l}$ of final volume.

For all qRT-PCR reactions, the qPCR-Core-Kit for SYBR-Green I No Rox (Eurogentec, Cologne, Germany) and StepOnePlus Real-Time-PCR devices (Applied Biosystems, Darmstadt, Germany) were used. Standard curves for quantifying copy numbers were generated using respective products from former test PCRs, which were extracted from agarose gel after electrophoresis. The purified template cDNA allowed serial dilutions for different copy numbers $\left(10^{1}\right.$ to $\left.10^{7}\right)$. For absolute quantification, the copy number of target genes was extrapolated from the standard curve equation.

For normalization, GeNorm software algorithm (28) selected HPRT1 (hypoxanthine phosphoribosyltransferase 1), TUBA1A (tubulin $\alpha$ 1a) and RPS18 (ribosomal protein S18) as the most suitable out of four housekeeping genes (HPRT1, $T U B A 1, R P S 18, G A P D H)$. Primer pairs for qRT-PCR were $B O R I S$ forward, GAGCGGGACCATGAAAATACATA and BORIS reverse, ATATGCACACGTAGGTCGCTTTT (114 bp, $62^{\circ} \mathrm{C}$ annealing temperature); TSHZ1 forward, CGCAGTCAAACTGCACCTTA and TSHZ1 reverse, CTCG ACGACGTTTAGTGCAA (134 bp, $\left.61.5^{\circ} \mathrm{C}\right)$; RPS18 forward, AGTTCCAGCATATTTTGCGAGTA and $R P S 18$ reverse, TTTCCATCCTTTACATCCTTCTG $\left(259 \mathrm{bp}, 61.4^{\circ} \mathrm{C}\right)$; HPRT1 forward, GACCAGTCAACAGGGGACAT and HPRT1 reverse, CCTGACCAAGGAAAGCAAAG (132 bp, $\left.59^{\circ} \mathrm{C}\right) ; T U B A 1$ forward, GATTATGGCAAGAAGTCCAAGC and $T U B A 1$ reverse, TACCATGAAGGCACAATCAGAG (135 bp, $\left.59^{\circ} \mathrm{C}\right)$.

Statistical analysis. Statistical calculations were carried out using the Kruskal-Wallis test and Dunn's multiple comparison test. Differences with a $\mathrm{p}<0.05$ were considered as significant.

Western blot analysis. Of each tissue sample, 25-40 mg were homogenized (T25 Basic, IKA Labortechnik, Staufen, Germany) in $1 \mathrm{ml}$ lysis buffer (50 mM Tris $\mathrm{pH} 7.4,300 \mathrm{mM}$ $\mathrm{NaCl}, 10$ mM EDTA, 1\% NP40, 0.1\% Triton X-100, 0.1\% SDS, protease inhibitor cocktail Complete ${ }^{\circledR}$ (Roche, Mannheim, Germany). Samples were incubated at $4^{\circ} \mathrm{C}$ for $2 \mathrm{~h}$ and centrifuged with $16,000 \mathrm{~g}$ for $1 \mathrm{~h}$. Protein concentration of the supernatant was analyzed using BCA assays (Pierce Biotechnology, Rockford, USA) and aliquots were stored until usage at $-80^{\circ} \mathrm{C}$.
After denaturation $\left(3 \mathrm{~min}\right.$ at $90^{\circ} \mathrm{C}$ with $\mathrm{SDS}$ loading buffer including mercaptoethanol), $50 \mu \mathrm{g}$ of lysate was applied to each lane, separated by SDS polyacrylamide gel electrophoresis (SDS-PAGE) on $10 \%$ gels and transferred to nitrocellulose membranes (Protran-BA-83, Schleicher \& Schuell,Dassel,Germany). Ponceau S staining served as additional loading control. For protein detection, primary antibodies against BORIS (1:2000, rabbit polyclonal ab18337, Abcam Inc., UK, Cambridge) and TSHZ1 (1:200, rabbit polyclonal M5943, Sigma, Taufkirchen, Germany) diluted in RotiBlock (Carl Roth, Karlsruhe, Germany) were used. HRP-conjugated $\mathrm{F}\left(\mathrm{ab}^{\prime}\right) 2$ goat anti-rabbit $\operatorname{IgG}(\mathrm{H}+\mathrm{L})$ secondary antibodies (KPL, Gaithersburg, USA), diluted 1:5000 in RotiBlock, were applied for $60 \mathrm{~min}$, followed by peroxidase substrate Supersignal West Dura (Thermo Scientific, Bonn, Germany) for $5 \mathrm{~min}$. Finally, signals were detected by the LumiImager-F1 (Roche, Mannheim, Germany).

Immunofluorescence staining. Immunofluorescence staining of freshly prepared $5 \mu \mathrm{m}$ cryosections was performed using an affinity-purified rabbit polyclonal TSHZ1 antibody (1:200, M5943, Sigma, Taufkirchen, Germany) and an affinitypurified rabbit polyclonal BORIS antibody (1:200, ab18337, Abcam Inc., Cambridge, UK). To differentiate expression of BORIS and TSHZ1 in endothelial and stromal mesenchymal cells, co-localization studies with the mouse anti-CD31 antibody as an endothelial marker (1:40,JC-70A, Dako, Germany) and the mouse anti-prolyl-hydroxylase $\beta$ antibody as a marker of mesenchymal stromal cells (1:400, clone 3-2B12, Acris, Hiddenhausen, Germany) were performed, respectively. DAPI (Vector Laboratories, Burlingame, CA, USA) was used for nuclear counterstaining. Sections were analyzed and photographed with the APOTOME technique (Zeiss, Jena, Germany).

\section{Results}

Detection of BORIS and TSHZ1 gains in JAs. In order to identify small genome aberrations specific for juvenile angiofibromas, genome-wide copy number profiling using a $100 \mathrm{~K}$ SNP array with a resolution down to $200 \mathrm{~kb}$ was performed on $14 \mathrm{JA}$ samples and peripheral blood from two of the $14 \mathrm{JA}$ patients (set I). Compared to the blood samples, where only copy number variations common in the general population were detected, a large number of unusual aberrations was found in each of the 14 investigated tumors (data not shown). By using the strict evaluation algorithm described above, 30 duplications on autosomal chromosomes were identified. Of those, four were detected in 7/14 and two were found in 8/14 tumor samples (Table I, Fig. 1). Among these interesting candidate genes for JA-specific gains, two with possible implications for JA biology were selected for further analysis: BORIS (20q13.3), which was amplified in 8/14 JAs, was chosen because its gene product is known as a cancer-testis antigen involved in cell proliferation and epigenetic dysregulation of cancers (18). Second, TSHZ1 (18q22.3), which showed gains in $7 / 14$ tumor specimens, emerged as an attractive candidate gene with respect to our hypothesis of JAs as vascular malformations of the first branchial arch artery $(4,7,29)$.

Quantitative genomic PCR from 12 of the 14 JA tumors and six peripheral blood samples from healthy controls (set I) 
Table I. JA-specific chromosomal alterations detected by the 100K SNP DNA microarray.

\begin{tabular}{|c|c|c|c|c|}
\hline Location & rs-number & SNP & Location in bp & Affected tumors \\
\hline $1 \mathrm{p} 33$ & rs10489770-rs1002378 & SNP_A-1672946-SNP_A-1699173 & $46580180-47317900$ & Gains in 3 out of 14 \\
\hline $1 \mathrm{p} 31.1$ & rs10493484-rs2125775 & SNP_A-1653047-SNP_A-1688718 & $71474040-71474050$ & Gains in 5 out of 14 \\
\hline $1 \mathrm{q} 23.3$ & rs10494435-rs10494442 & SNP_A-1702658-SNP_A-1650800 & $163442185-163521850$ & Gains in 3 out of 14 \\
\hline $1 q 44$ & rs2365082-rs2362932 & SNP_A-1732549-SNP_A-1649226 & $243398800-243980910$ & Gains in 3 out of 14 \\
\hline $2 \mathrm{p} 25.1$ & rs7570654-rs 10495548 & SNP_A-1737596-SNP_A-1661317 & $6874422-7054890$ & Gains in 4 out of 14 \\
\hline $2 \mathrm{q} 22.3$ & $-r s 274844$ & SNP_A-1710906-SNP_A-1714655 & -145634550 & Gains in 3 out of 14 \\
\hline $3 \mathrm{p} 22.1$ & rs2371319-rs422623 & SNP_A-1699128-SNP_A-1696672 & $40701945-41098520$ & Gains in 7 out of 14 \\
\hline $3 \mathrm{p} 11.1$ & rs1483489-rs3946805 & SNP_A-1743831-SNP_A-1655924 & $88963955-89399070$ & Gains in 5 out of 14 \\
\hline $3 q 26.32$ & rs9290637-rs4076914 & SNP_A-1755631-SNP_A-1657013 & $179374400-180154385$ & Gains in 3 out of 14 \\
\hline $7 \mathrm{p} 12.3$ & rs2768419-rs10240495 & SNP_A-1755341-SNP_A-1665150 & $49502965-49791960$ & Gains in 8 out of 14 \\
\hline $8 q 11.21$ & rs1372062-rs341817 & SNP_A-1686924-SNP_A-1664173 & $50028400-50186155$ & Gains in 3 out of 14 \\
\hline $8 q 22.1$ & rs6991577-rs7828725 & SNP_A-1654545-SNP_A-1676299 & $93948600-94208095$ & Gains in 3 out of 14 \\
\hline $8 \mathrm{q} 22.1$ & rs10504974-rs1872011 & SNP_A-1715057-SNP_A-1657906 & $98591510-98888600$ & Gains in 3 out of 14 \\
\hline $8 q 24.13$ & rs4128469-rs723231 & SNP_A-1753969-SNP_A-1673612 & $125548650-126018235$ & Gains in 4 out of 14 \\
\hline $9 \mathrm{p} 21.3$ & rs1329036-rs10491795 & SNP_A-1653471-SNP_A-1685579 & $23819910-24103907$ & Gains in 4 out of 14 \\
\hline $9 q 31.2$ & rs4978698-rs4259479 & SNP_A-1741597-SNP_A-1652845 & $110349970-110564480$ & Gains in 3 out of 14 \\
\hline $9 \mathrm{q} 33.1$ & rs7032395-rs 10513300 & SNP_A-1697364-SNP_A-1701826 & $118867780-119170030$ & Gains in 3 out of 14 \\
\hline $9 q 34.13$ & rs10512414-rs4128956 & SNP_A-1717146-SNP_A-1721113 & $133325225-133808330$ & Gains in 4 out of 14 \\
\hline $10 q 22.1$ & rs1417909-rs7918636 & SNP_A-1723364-SNP_A-1645168 & $72592370-72885535$ & Gains in 3 out of 14 \\
\hline $10 q 26.13$ & -rs2292623 & SNP_A-1672547-SNP_A-1679262 & & Gains in 7 out of 14 \\
\hline $11 \mathrm{q} 25$ & rs1793821-rs1355982 & SNP_A-1754058-SNP_A-1736383 & $130525080-130853300$ & Gains in 7 out of 14 \\
\hline $12 \mathrm{q} 21.33$ & rs1504143-rs10492233 & SNP_A-1657346-SNP_A-1713503 & $89744900-89938450$ & Gains in 5 out of 14 \\
\hline $12 q 23.2$ & rs825041-rs10507135 & SNP_A-1665482-SNP_A-1703736 & $100485005-100853956$ & Gains in 3 out of 14 \\
\hline $13 q .34$ & rs496916-rs718886 & SNP_A-1673670-SNP_A-1666004 & $109649015-110044380$ & Gains in 4 out of 14 \\
\hline $14 q 32.2-q 32.31$ & rs736319-rs2152369 & SNP_A-1670897-SNP_A-1716060 & $99959035-100696370$ & Gains in 3 out of 14 \\
\hline $15 q 21.1$ & rs2460620-rs8039174 & SNP_A-1662257-SNP_A-1727759 & $44085750-44485440$ & Gains in 4 out of 14 \\
\hline $16 q 23.1$ & rs1364095-rs1423815 & SNP_A-1693240-SNP_A-1708947 & $78123651-78194350$ & Gains in 3 out of 14 \\
\hline $18 \mathrm{q} 12.2$ & rs10502699-rs10502705 & SNP_A-1704978-SNP_A-1699983 & $34337732-34477370$ & Gains in 4 out of 14 \\
\hline $18 q 22.3$ & rs6566067-rs9319680 & SNP_A-1746537-SNP_A-1647465 & $71104940-71260335$ & Gains in 7 out of 14 \\
\hline $20 q 13.3$ & rs911901-rs326832 & SNP_A-1758638-SNP_A-1698862 & $55385594-55952451$ & Gains in 8 out of 14 \\
\hline
\end{tabular}

Data associated with the chromosomal localization of TSHZ1 (18q22.3) and BORIS (20q13.3) is bold-typed.

was used for validation of the results obtained with the $100 \mathrm{~K}$ SNP assay. To rule out a common SNP polymorphism for the selected genes, control DNA from blood of healthy donors $(n=6)$ was studied first (Fig. 2, control 1-6). Here, qPCR of genomic DNA for both candidate genes produced $\Delta \Delta \mathrm{Ct}$ values ranging from 0.97 to 1.08 , indicating a diploid genotype. A $\Delta \Delta \mathrm{Ct}$ value $>1.2$ confirmed $B O R I S$ gene duplication in five of the six tumors which had shown BORIS gains in the 100K SNP array (Fig. 2A, cases 7-12). On the contrary, $\Delta \Delta \mathrm{Ct}$ values were $<1.2$ for $6 / 6 \mathrm{JA}$ specimens lacking an amplification of BORIS in the microarray (Fig. 2A, cases 1-6). Duplication of the TSHZ1 gene was confirmed in 6/6 JAs by a $\Delta \Delta \mathrm{Ct}$ value $>1.2$ in qPCR (Fig. 2B, cases 7-12). Each of the six tumors without a detectable gain in TSHZ1 in the 100K SNP array showed a $\Delta \Delta \mathrm{Ct}$ value $<1.2$, which is in agreement with a diploid genotype (Fig. 2B, cases 1-6).

Expression of BORIS and TSHZ1 gene products in JAs. As the amount of tumor tissue from the 14 JA samples of set I was not sufficient for further investigations, a different set of $15 \mathrm{JAs}$ and 11 nasal mucosa specimens (set II) was used for expression analysis of BORIS and TSHZ1 on the mRNA and protein level.

For both genes, mRNA copy numbers were significantly higher in JAs compared to the subepithelial stroma of nasal mucosa (BORIS, p<0.001; TSHZ1, p<0.05) (Fig. 3). In order to investigate whether the up-regulation of BORIS and TSHZ1 transcripts in JAs was reflected on the translational level, protein samples available from eight JAs and six nasal mucosa specimens (set II) were objected to Western blotting. A signal of about $83 \mathrm{kD}$ was detected for BORIS protein in $8 / 8 \mathrm{JAs}$ and in 3/6 inferior turbinates (Fig. 4). For TSHZ1, a band at approximately $115 \mathrm{kD}$ was observed for $8 / 8 \mathrm{JAs}$ and $6 / 6$ nasal mucosa samples (Fig. 4). On visual inspection, TSZH1 signal intensity was lower for all NM compared to JA specimens.

In order to determine the localization of BORIS and TSHZ1 proteins in juvenile angiofibromas, immunofluorescence analyses of JA cryosections were performed. A dot-like 


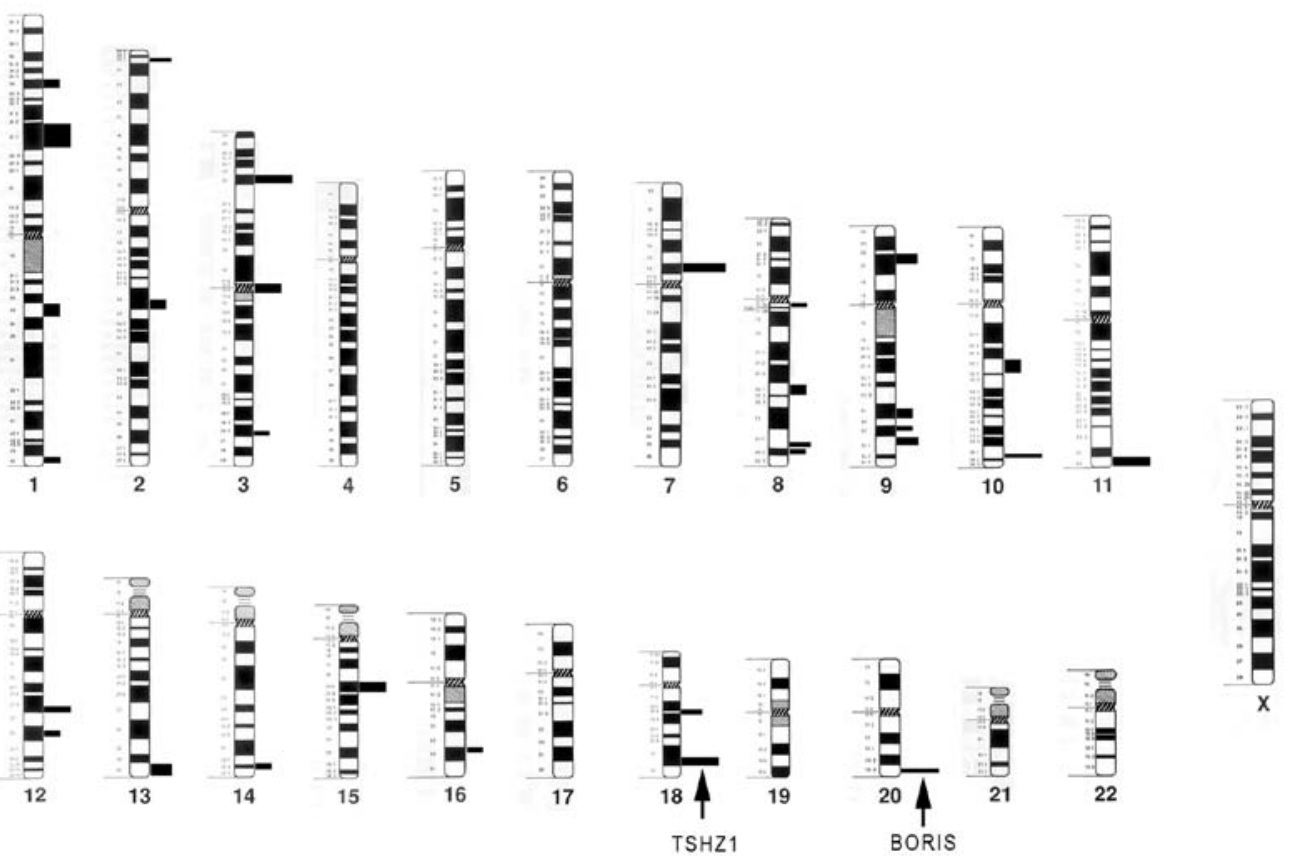

Figure 1. Ideogram of the duplications detected in $14 \mathrm{JAs}$ using a 100K SNP array with genomic smoothening algorithm (GSA)-p-values $>+2.5$ for duplications (at least 3 SNPs $>+5$ ) and $<-2.5$ for deletions (at least 3 SNPs $<-5$ ). The widths of the bars indicate the number of angiofibromas in which previously unknown gains were detected.
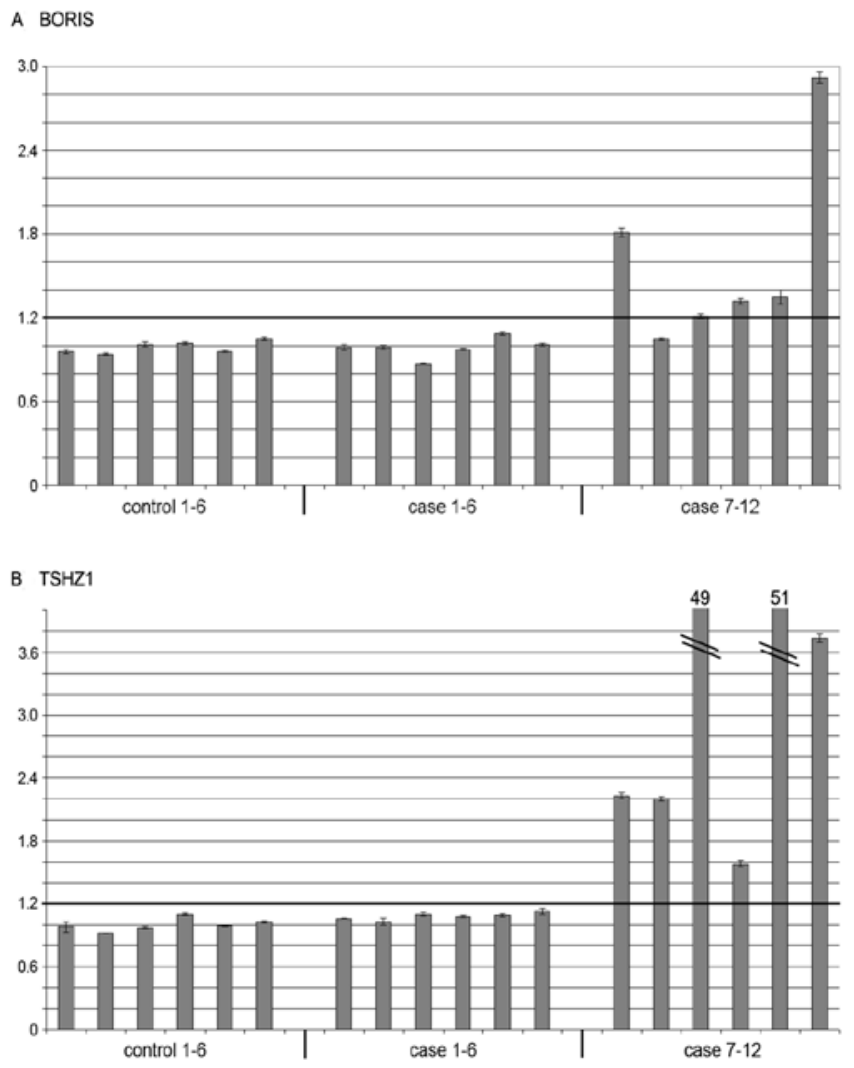

Figure 2. Results of genomic qPCR for BORIS (A) and TSHZ1 (B). $\Delta \Delta \mathrm{Ct}$ values from 0.8 to 1.2 (y-axis) were judged as diploid (normal) genotype, whereas a $\Delta \Delta \mathrm{Ct}$ value $>1.2$ was interpreted as an amplification. Blood samples from 6 healthy individuals (controls 1-6) displayed $\Delta \Delta \mathrm{Ct}$ values $<1.2$ for BORIS and TSHZ1. (A) $\Delta \Delta \mathrm{Ct}$ values were $<1.2$ for $6 / 6 \mathrm{JAs}$ without gains for BORIS in the 100K SNP array (cases 1-6), whereas 5/6 JAs with BORIS duplication in the microarray showed $\Delta \Delta \mathrm{Ct}$ values $>1.2$ (cases 7-12). (B) $\Delta \Delta \mathrm{Ct}$ values $<1.2$ were detected for $6 / 6 \mathrm{JAs}$ without gains for TSZH1 in the $100 \mathrm{~K}$ SNP array (cases 1-6). 6/6 JAs with TSHZ1 amplifications in the microarray displayed $\Delta \Delta \mathrm{Ct}$ values $>1.2$ (cases 7-12).
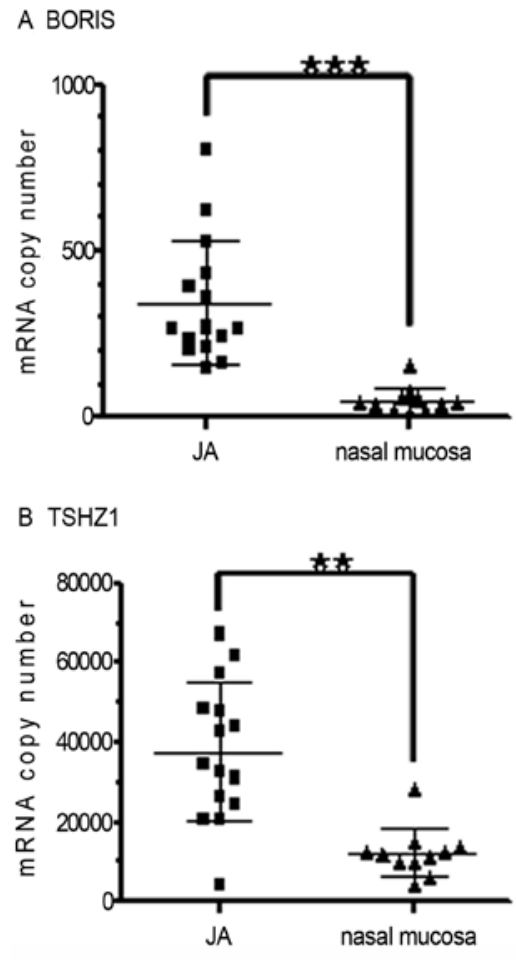

Figure 3. BORIS (A) and TSHZ1 (B) mRNA expression in JAs compared to nasal mucosa. qRT PCR revealed a significant up-regulation of BORIS $\left({ }^{* * *} \mathrm{p}<0.001\right)$ and TSHZ1 transcripts $\left.{ }^{* * *} \mathrm{p}<0.05\right)$ in JAs compared to the subepithelial stroma of nasal mucosa from control persons.

predominantly nuclear expression was detected for BORIS (Fig. 5A, red, arrow). Co-staining with the endothelial marker CD31 (green) revealed BORIS protein in the cell nuclei of endothelial (EC) and perivascular cells surrounding 


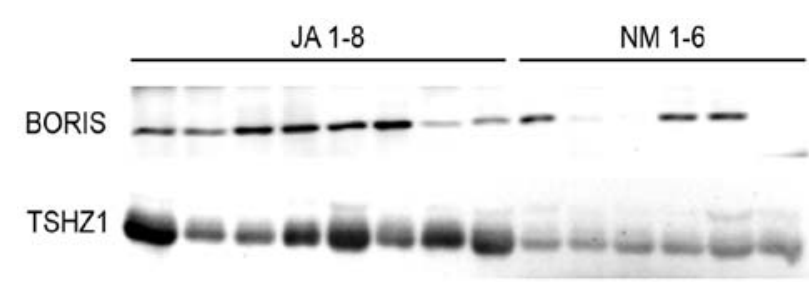

Figure 4. Western blot analysis of BORIS and TSHZ1 protein expression in 8 JAs (JA 1-8) and 6 specimens of nasal mucosa (NM 1-6). A signal for BORIS was detected at $83 \mathrm{kD}$ for $8 / 8 \mathrm{JAs}$ and $3 / 6 \mathrm{NM}$ samples. TSHZ1 protein was identified at $115 \mathrm{kD}$ in $8 / 8 \mathrm{JAs}$, a faint signal for TSHZ1 was visible in $6 / 6$ NM specimens.

blood vessels (V) and in mesenchymal cells (MC) (Fig. 5A). Visualization of the mesenchymal stromal cell marker protein prolyl-hydoxylase $\beta$ (green) identified BORIS (red, arrow) also in the cytoplasm of JA stromal cells (Fig. 5B, yellow signal). TSHZ1 showed a punctuated, mainly cytoplasmatic staining pattern (Fig. 5C and D; red, arrow). Most prominent staining for TSHZ1 around JA blood vessels (V) was seen in perivascular cells (Fig. 5C, PC). Dot-like TSHZ1 signals were also detected in endothelial cells (Fig. 5D, EC), and co-localization (yellow) of TSHZ1 (red) with prolyl-hydoxylase $\beta$ (green) confirmed expression of TSHZ1 in the cytoplasm of stromal cells (Fig. 5D, MC).
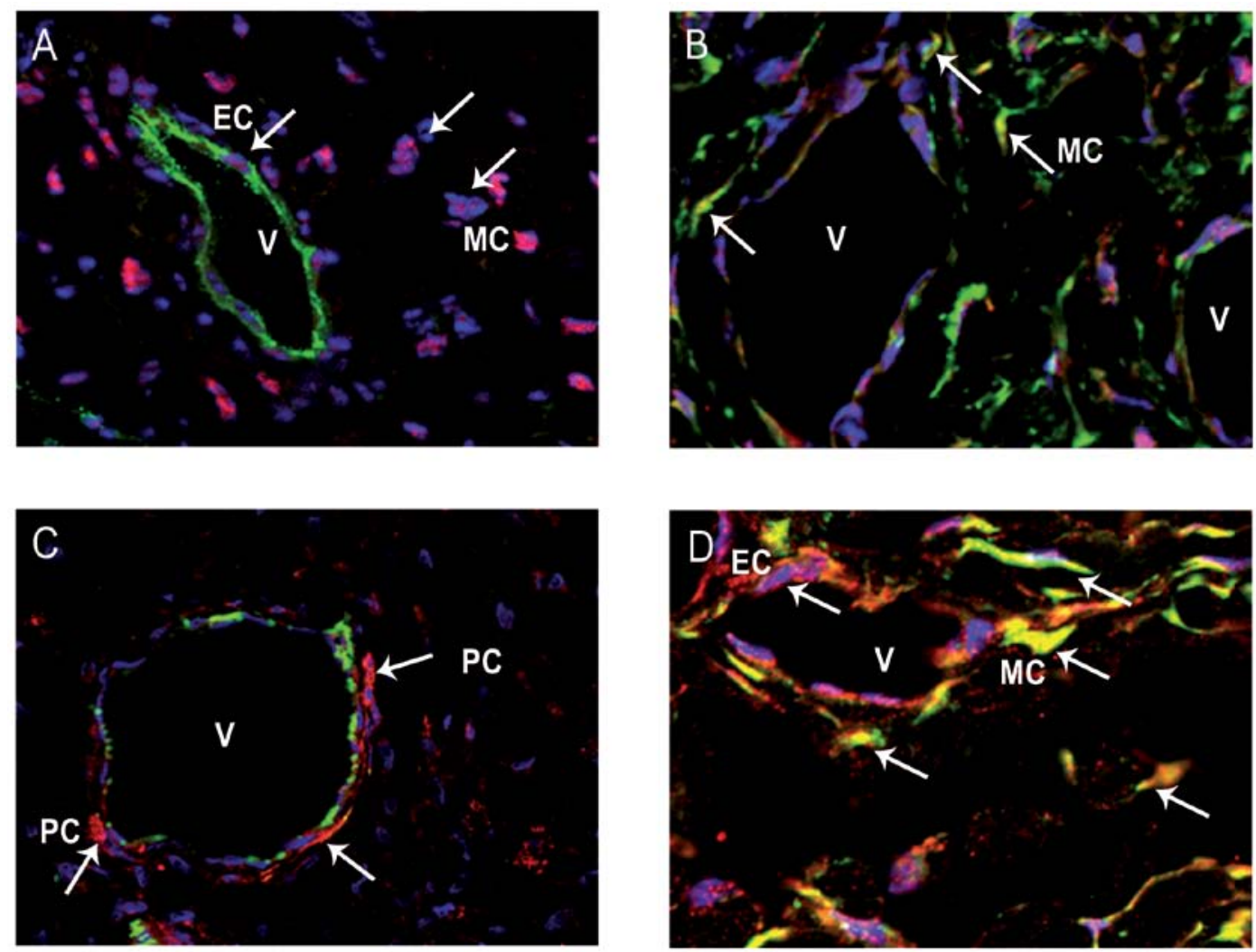

Figure 5. Immunostaining of BORIS and TSHZ1 protein in cryosections of JAs. Cell nuclei were counterstained with DAPI (blue). (A) Endothelial cells were stained with CD31 (green). BORIS (red, arrow) was detected in the cell nuclei of endothelial (EC, green), perivascular and mesenchymal cells (MC). The lumen of the vessel is indicated by 'V'. (B) An antibody against prolyl-hydroxylase $\beta$ (green) was used as a cytoplasmic marker for mesenchymal stroma cells. BORIS protein (red, arrow) was co-localized with prolyl-hydoxylase $\beta$ (green) in the cytoplasm (yellow signal) of mesenchymal cells (MC). (C) Co-staining of JA vessels with an antibody against TSHZ1 (red, arrow) and the endothelial marker CD31 (green) visualized TSHZ1 protein in perivascular cells (PC). (D) TSHZ1 (red, arrow) was detected in the cell nuclei of endothelial cells (EC) and co-localizes (yellow signal) with prolyl-hydoxylase $\beta$ (green) in the cytoplasm of mesenchymal cells (MC). 
together, the genetic alterations on chromosome $20 q$ and $16 q$ observed in JAs in our present and previous studies suggest a role of $\mathrm{CTCF} / \mathrm{BORIS}$ competition in the pathogenesis of this unique tumor.

BORIS belongs to the group of cancer-testis antigens (CTA), which are normally almost exclusively found in testis, but are abnormally activated in cancer cells (18). As testis is an immune-privileged organ, CTAs may be considered as non-self antigens capable of eliciting strong anti-cancer immunity. Due to their immunogenicity and tumor-restricted expression, several CTAs have been used in recent years to develop targeted anti-tumor vaccines (21). BORIS is not only a cancer-testis antigen itself, but has also been associated with derepression of other CTAs including NY-ESO-1 and MAGE-A1 $(30,31)$. Therefore, BORIS has become an attractive candidate antigen for tumor vaccination. Recent studies showed that a DNA vaccination against BORIS inhibited tumor growth and prolonged survival in a mouse model of mammary adenocarcinoma $(32,33)$.

In the present study, a significant up-regulation of BORIS transcripts was observed in JAs compared to the subepithelial stroma of nasal mucosa (Fig. 3A). Low-level expression of BORIS mRNA in nasal mucosa from healthy individuals is in line with the previous detection of small amounts of BORIS mRNA in pancreas, prostate, thymus and kidney. Consequently, BORIS is regarded as a 'differentially expressed CT gene', whose transcripts can be detected in normal tissues apart from testis, albeit at a lower level (21). Observation of BORIS protein in some control tissue samples in two studies investigating BORIS expression in breast cancer $(34,35)$ fits with this concept. Identification of BORIS protein in $3 / 6$ nasal mucosa samples compared to $8 / 8 \mathrm{JAs}$ in the present study (Fig. 4) is in line with the notion that BORIS can indeed be found in non-tumor tissues. Due to the heterogeneity of native nasal mucosa with a high proportion of glands in the epithelium, a quantitative interpretation of protein signals in Western blots comparing NM and JA is not advisable. Furthermore, it has to be considered that only a small number of JAs $(n=8)$ was available for this study. As JA is such a rare tumor, multi-center studies would be necessary in the future in order to obtain the exact relation of BORIS protein expression in JA versus nasal mucosa samples. In this context, a potential impact of BORIS expression in normal tissue on the immunogenic potential of this CTA needs further evaluation (21). Nevertheless, the option of designing an anti-JA tumor vaccine in the future is of high clinical interest as currently no effective drug treatment is available for this fibrovascular tumor. Anti-tumor vaccination would offer an attractive opportunity to induce tumor shrinkage of advanced JAs prior to operation.

Potential impact of BORIS on JA cell proliferation, hormone signaling and epigenetic dysregulation. The ubiquitously expressed transcription factor CTCF regulates numerous genes associated with cell cycle regulation and cell proliferation. Aberrant expression of BORIS is suggested to compete with CTCF in regulation of its target genes (18). Studies from immature B cells have shown that CTCF controls both MYC and $M D M 2$ oncogenes (36). Gains for both genes have been identified for JAs in our previous studies $(15,16)$. Detection of BORIS in JAs in the present study raises the question whether it activates $M Y C$ and $M D M 2$ in this fibrovascular tumor. Recently, Nguyen et al (37) observed binding of BORIS protein to the $M Y C$ promoter and down-regulation of $M Y C$ transcripts induced by small hairpin (sh) RNA knockdown of BORIS in colon carcinoma cells suggesting that BORIS enhances MYC expression in cancer cells. Further investigations will be necessary to determine whether the molecular mechanisms of BORIS/MYC interaction described above can be verified for JAs and whether BORIS interferes with CTCF-associated regulation of MDM2.

Beside its impact on cell-proliferation associated genes, BORIS has been reported to interfere with hormone receptors in human cancers. A recent study did not only identify BORIS protein in $70 \%$ of the analyzed breast cancer specimens, but also correlated high levels of BORIS with high levels of estrogen receptor (ER) and progesterone receptor (PR) proteins. Moreover, BORIS was shown to activate the promotors of both ER and PR genes in reporter assays (34). Concerning JAs, expression and function of sex steroid receptors are still controversial issues (38-40). A recent study detected $\mathrm{P} 450$ aromatase, which is able to convert androgens into estrogens, in 5/5 analyzed JAs (41). Future investigations into a possible role of BORIS in hormone-dependent gene regulation in JAs may shed light on the interplay of estrogen, progesteron and androgen in this tumor, which almost exclusively afflicts adolescent males.

CTCF interacts with chromatin insulators/boundaries, which partition the genome into distinct functional domains preventing long-distance communication of cis regulatory elements, e.g. promotors, with enhancers/silencers (19). This role of $\mathrm{CTCF}$ in epigenetic regulation has extensively been studied for the H19/IGF2 imprinting control region (ICR). IGFs (insulin like growth factors) have been suggested to be involved in tumorigenesis by promoting cell growth and division (42). CTCF is only able to bind to the unmethylated ICR on the maternal allele, thus blocking the communication between the $I G F 2$ promotor and a downstream enhancer resulting in silencing of $I G F 2$. Methylation of the maternal $H 19 / I G F 2$ ICR in cancer cells abrogates binding of CTCF leading to derepression of IGF2 gene expression (19). In contrast to its paralog CTCF, BORIS is a methylation-independent DNA-binding protein that preferentially binds to the methylated H19/IGF2 ICR (43). Due to these observations, BORIS is supposed to play a central role in epigenetic dysregulation including loss of imprinting (LOI), which is a common feature in many cancers. LOI for the IGF2 gene (17) and up-regulation of IGF2 mRNA (44) have also been observed in JAs previously. Further investigations will be necessary to elucidate whether BORIS is involved in epigenetic dysregulation of H19/IGF2 and other genes in JAs.

Expression of TSHZ1 in JAs. In mice, Tshzl gene products have been located to neural crest-derived mesenchymal cells of the first and second branchial arches (6) and have been associated with development of the soft palate and the middle ear (7). Here, we are the first to describe amplification of the human ortholog TSHZ1 in JAs (Figs. 1 and 2) resulting in an up-regulation of $T S H Z 1$ transcripts compared to nasal mucosa (Fig. 3B). Western blotting revealed TSHZ1 protein expression in $8 / 8 \mathrm{JAs}$, while only a faint protein signal was detected in 6/6 nasal mucosa specimens (Fig. 4). The pivotal role of Tshz1 
in the development of the soft palate and middle ear (7) offers an explanation for low-level expression of TSHZ1 in nasal mucosa, which forms the epithelial surface of both the soft palate and the middle ear. Up-regulation of TSHZ1 mRNA (Fig. 3B) and localization of TSHZ1 protein in perivascular and mesenchymal cells in JAs (Fig. 5C and D) are interesting findings concerning the origin of JAs: anatomical studies on blood supply (4) of JAs and the vascular architecture of this fibrovascular tumor (5) have given rise to the hypothesis of JAs evolving from remnants of the first branchial arch artery. Identification of the developmental regulatory factor TSHZ1, whose mouse ortholog is found in mesenchymal cells of the first branchial arch, in JAs adds further evidence to this hypothesis on the molecular level.

Possible roles of TSHZ1 in JA tumorigenesis. The Drosophila ortholog of TSHZ1, tsh (teashirt), is required for maintenance of the Wg (wingless) pathway, which corresponds to the Wnt signaling cascade in vertebrates $(45,46)$. The Wnt pathway is not only essential for body plan formation during embryonal development (47), but is also involved in tumorigenesis of many cancers (48). Considering previous evidence for a role of Wnt signaling in JAs (12), a comparison between the Wg and Wnt pathways regarding the role of $t$ sh and TSHZl may offer new insights into tumor biology of JAs: in Drosophila, Tsh binds to and stabilizes the transcription factor Armadillo (Arm), an analog to $\beta$-catenin in vertebrates $(45,49)$. Furthermore, a Xenopus laevis ortholog of Tsh, XTsh3, has been shown to enhance Wnt signaling by binding to $\beta$-catenin and enhancing $\beta$-catenin levels in the nucleus (50). In JAs, frequent activating $\beta$-catenin mutations have been detected previously (10). These observations raise the question whether TSHZ1 is a binding partner of $\beta$-catenin in JAs capable of shaping Wnt signaling in this rare fibrovascular tumor.

Beside their role in embryonal development, gene products of teashirt orthologs also have to be considered as cancer antigens. TSHZ1 antigen was identified in a human cDNA expression library derived from human colon cancers and is therefore also known as SDCCAG 33 (serologically defined colon cancer antigen 33) or NY-CO-33 (21). Reactivation of developmental signaling cascades like the Wnt pathway is a common feature in many tumors.

In conclusion, the present study shows that molecular karyotyping using an SNP array is a suitable and reliable approach for detecting genetic alterations in JAs. Using this technique, the products of two regulatory genes were identified as novel tumor markers of JAs: BORIS, which is normally involved in imprinting during male sperm cell development and the colon cancer antigen TSHZ1, whose mouse ortholog is found in derivates of the first branchial arch. Future investigations will be needed to elucidate the underlying molecular mechanisms and to unravel the functional implications of BORIS and TSHZ1 in this unique fibrovascular tumor.

\section{Acknowledgements}

This study was supported by a grant of the Marohn Foundation at the University of Erlangen-Nuremberg to B. Schick. The authors thank Renate Schäfer and Claudia Bevacqua for excellent technical assistance.

\section{References}

1. Schick B and Kahle G: Radiological findings in angiofibroma. Acta Radiol 41: 585-593, 2000.

2. Eivazi B, Ardelean M, Bäumler W, Berlien HP, Cremer H, Elluru R, Koltai P, Olofsson J, Richter G, Schick B and Werner JA: Update on hemangiomas and vascular malformations of the head and neck. Eur Arch Otorhinolaryngol 266: 187-197, 2009.

3. Beham A, Beham-Schmid C, Regauer S, Aubock L and Stammberger H: Nasopharyngeal angiofibromas: true neoplasm or vascular malformation. Adv Anat Pathol 7: 36-46, 2000.

4. Schick B, Plinkert PK and Prescher A: Aetiology of angiofibromas: reflection on their specific vascular component. Laryngorhinootol 81: 280-284, 2002.

5. Starlinger V, Wendler O, Gramann M and Schick B: Laminin expression in juvenile angiofibromas indicates vessel's early developmental stage. Acta Otolaryngol 127: 1310-1315, 2007.

6. Long Q, Park BK and Ekker M: Expression and regulation of mouse Mtsh1 during limb and branchial arch development. Dev Dyn 222: 308-312, 2001.

7. Coré N, Caubit X, Metchat A, Boned A, Djabali M and Fasano L: Tshz 1 is required to axial skeleton, soft palate and middle ear development in mice. Dev Biol 308: 407-420, 2007.

8. Guertl B, Beham A, Zechner R, Stammberger H and Hoefler G: Nasopharyngeal angiofibroma: an APC-gene-associated tumor? Hum Pathol 31: 1411-1413, 2000.

9. Komiya $\mathrm{Y}$ and Habas R: Wnt signal transduction pathways. Organogenesis 4: 68-75, 2008.

10. Abraham SC, Montgomery EA, Giardiello FM and Wu TT: Frequent beta-catenin mutations in juvenile nasopharyngeal angiofibromas. Am J Pathol 158: 1073-1078, 2001.

11. Schick B, Rippel C, Brunner C, Jung V, Plinkert PK and Urbschat S: Numerical sex chromosome aberrations in juvenile angiofibromas: genetic evidence for an androgen-dependent tumor? Oncol Rep 10: 1251-1255, 2003.

12. Coutinho-Camillo CM, Brentani MM and Nagai MA: Genetic alterations in juvenile nasopharyngeal angiofibromas. Head Neck 30: 390-400, 2008.

13. Schick B, Brunner C, Praetorius M, Plinkert PK and Urbschat S: First evidence of genetic imbalances in angiofibromas. Laryngoscope 112: 397-401, 2002.

14. Brunner C, Urbschat S, Jung V, Praetorius M, Schick B and Plinkert PK: Chromosomal alterations in juvenile angiofibromas. HNO 51: 981-985, 2003.

15. Schick B, Wemmert S, Bechtel U, Nicolai P, Hofmann T, Golabek W and Urbschat S: Comprehensive genomic analysis identifies MDM2 and AURKA as novel amplified genes in juvenile angiofibromas. Head Neck 29: 479-487, 2007.

16. Schick B, Wemmert S, Jung V, Steudel WI, Montenarh M and Urbschat S: Genetic heterogeneity of the MYC oncogene in advanced juvenile angiofibromas. Cancer Genet Cytogenet 164: 25-31, 2006.

17. Coutinho-Camillo CM, Brentani MM, Butugan O, Torloni $\mathrm{H}$ and Nagai MA: Relaxation of imprinting of IGFII gene in juvenile nasopharyngeal angiofibromas. Diagn Mol Pathol 12: 57-62, 2003.

18. Klenova EM, Morse HC, Ohlsson R and Lobanenkov VV: The novel BORIS + CTCF gene family is uniquely involved in the epigenetics of normal biology and cancer. Semin Cancer Biol 12: 399-314, 2002.

19. Ohlsson R, Renkawitz R and Lobanenkov V: CTCF is a uniquely versatile transcription regulator linked to epigenetics and disease. Trends Genet 17: 520-527, 2001.

20. Loukinov DI, Pugacheva E, Vatolin S, et al: BORIS, a novel male germ-line specific protein associated with epigenetic reprogramming events, shares the same 11-zinc-finger domain with CTCF, the insulator protein involved in reading imprinting marks in the soma. Proc Natl Acad Sci 99: 6806-6811, 2002.

21. Scanlan MJ, Simpson AJ and Old LJ: The cancer/testis genes: review, standardization and commentary. Cancer Immun 4: 1-15, 2004.

22. Ellis NA, Kirchhoff T, Mitra N, et al: Localization of breast cancer susceptibility loci by genome-wide SNP linkage disequilibrium mapping. Genet Epidemiol 30: 48-61, 2006.

23. Andrews JC, Fisch U, Valavanis A, Aeppli U and Makek MS: The surgical management of extensive nasopharyngeal angiofibromas with the infratemporal fossa approach. Laryngoscope 99: 429-437, 1989. 
24. Hoyer J, Dreweke A, Becker C, et al: Molecular karyotyping in patients with mental retardation using 100K SNP arrays. J Med Genet 4: 629-636, 2007.

25. Thiel V, Herold J and Siddell SG: Long distance reverse-transcription PCR. Methods Mol Biol 192: 59-66, 2002.

26. Thiel CT, Kraus C, Rauch A, Ekici AB, Rautenstrauss B and Reis A: A new quantitative PCR multiplex assay for rapid analysis of chromosome 17p11.2-12 duplications and deletions leading to HMSN/HNPP. Eur J Hum Genet 11: 170-178, 2002.

27. Wendler O, Schaefer R and Schick B: Mast cells and T-lymphocytes in juvenile angiofibromas. Eur Arch Otorhinolaryngol 264: 769-775, 2007.

28. Vandesompele J, De Preter K, Pattyn F, Poppe B, Van Roy N, De Paepe A and Speleman F: Accurate normalization of real-time quantitative RT-PCR data by geometric averaging of multiple internal control genes. Genome Biol 3: RESEARCH0034, 2002.

29. Caubit X, Coré N, Boned A, Kerridge S, Djabali M and Fasano L: Vertebrate orthologues of the Drosophila region-specific patterning gene teashirt. Mech Dev 91: 445-448, 2000.

30. Hong JA, Kang Y, Abdullaev Z, et al: Reciprocal binding of CTCF and BORIS to the NY-ESO-1 promoter coincides with derepression of this cancer-testis gene in lung cancer cells. Cancer Res 65: 7763-7774, 2005.

31. Vatolin S, Abdullaev Z, Pack SD, et al: Conditional expression of the CTCF-paralogous transcriptional factor BORIS in normal cells results in demethylation and depression of MAGE-A1 and reactivation of other cancer-testis genes. Cancer Res 65: 7751-7762, 2005.

32. Loukinov D, Ghochikyan A, Mkrtichyan M, Ichim TE, Lobanenkov VV, Cribbs DH and Agadjanyan MG: Antitumor efficacy of DNA vaccination to the epigenetically acting tumor promoting transcription factor BORIS and CD80 molecular adjuvant. J Cell Biochem 98: 1037-1043, 2006.

33. Mkrtichyan M, Ghochikyan A, Loukinov D, Davtyan H, Ichim TE, Cribbs DH, Lobanenkov VV and Agadjanyan MG: DNA, but not protein vaccine based on mutated BORIS antigen significantly inhibits tumor growth and prolongs survival of mice. Gene Ther 15: 61-64, 2008.

34. D'Arcy V, Pore N, Docquier F, Abdullaev ZK, Chernukhin I, Kita GX, Rai S, Smart M, Farrar D, Pack S, Lobanenkov V and Klenova E: BORIS, a paralogue of the transcription factor CTCF, is aberrantly expressed in breast tumours. Br J Cancer 98: 571-579, 2008

35. Zhou XC, Song CJ, Wang X, Tian Y and Jin BQ: Preparation of the monoclonal antibodies against human BORIS and the expression pattern of BORIS in normal and diseased human mammary tissue. Xi Bao Yu Fen Zi Mian Yi Xue Za Zhi 23 353-355, 2007.

36. Qi CF, Martensson A, Mattioli M, Dalla-Favera R Lobanenkov VV and Morse HC III: CTCF functions as a critical regulator of cell-cycle arrest and death after ligation of the $\mathrm{B}$ cell receptor on immature B cells. Proc Natl Acad Sci USA 100: 633-638, 2003.
37. Nguyen P, Bar-Sela G, Sun L, Bisht KS, Cui H, Kohn E, Feinberg AP and Gius D: BAT3 and SET1A form a complex with CTCFL/BORIS to modulate H3K4 histone dimethylation and gene expression. Mol Cell Biol 28: 6720-6729, 2008.

38. Hwang HC, Mills SE, Patterson K and Gown AM: Expression of androgen receptors in nasopharyngeal angiofibroma: an immunohistochemical study of 24 cases. Mod Pathol 11: 1122-1126, 1998.

39. Montag AG, Tretiakova M and Richardson M: Steroid hormone receptor expression in nasopharyngeal angiofibromas. Consistent expression of estrogen receptor beta. Am J Clin Pathol 125: 832-837, 2006.

40. Saylam G, Yücel OT, Sungur A and Onerci M: Proliferation, angiogenesis and hormonal markers in juvenile nasopharyngeal angiofibroma. Int J Pediatr Otorhinolaryngol 70: 227-234, 2006.

41. Schlauder SM, Knapp C, Steffensen TS and Bui MM: Aromatase may play a critical role in the pathogenesis of juvenile nasopharyngeal angiofibroma. Fetal Pediatr Pathol 28: 232-238, 2009.

42. Maki RG: Small is beautiful: insulin-like growth factors and their role in growth, development and cancer. J Clin Oncol 28 : 4985-4995, 2010

43. Nguyen P, Cui H, Bisht KS, Sun L, Patel K, Lee RS, Kugoh H, Oshimura M, Feinberg AP and Gius D: CTCFL/BORIS is a methylation-independent DNA-binding protein that preferentially binds to the paternal H19 differentially methylated region. Cancer Res 68: 5546-5551, 2008.

44. Nagai MA, Butugan O, Logullo A and Brentani MM: Expression of growth factors, proto-oncogenes, and p53 in nasopharyngeal angiofibromas. Laryngoscope 106: 190-195, 1996.

45. Gallet A, Erkner A, Charroux B, Fasano L and Kerridge S: Trunk-specific modulation of wingless signalling in Drosophila by teashirt binding to armadillo. Curr Biol 8: 893-902, 1998.

46. Manfroid I, Caubit X, Kerridge S and Fasano L: Three putative murine Teashirt orthologues specify trunk structures in Drosophila in the same way as the Drosophila teashirt gene. Development 131: 1065-1073, 2004.

47. Petersen CP and Reddien PW: Wnt signaling and the polarity of the primary body axis. Cell 139: 1056-1068, 2009.

48. Camilli TC and Weeraratna AT: Striking the target in Wnt-y conditions: intervening in Wnt signalling during cancer progression. Biochem Pharmacol 80: 702-711, 2010.

49. Gallet A, Angelats C, Erkner A, Charroux B, Fasano L and Kerridge S: The C-terminal domain of armadillo binds to hypophosphorylated teashirt to modulate wingless signalling in Drosophila. EMBO J 18: 2208-2217, 1999.

50. Onai $\mathrm{T}$, Matsuo-Takasaki $\mathrm{M}$, Inomata $\mathrm{H}$, Aramaki $\mathrm{T}$, Matsumura M, Yakura R, Sasai N and Sasai Y: XTsh3 is an essential enhancing factor of canonical Wnt signalling in Xenopus axial determination. EMBO J 26: 2350-2360, 2007. 